\title{
Plasma Levels of Soluble PD-L1 Correlate With Tumor Regression in Patients With Lung and Gastric Cancer Treated With Immune Checkpoint Inhibitors
}

\author{
KIYOHIRO ANDO ${ }^{1,2^{*}}$, KAZUYUKI HAMADA ${ }^{3 *}$, MAKOTO WATANABE ${ }^{1,2}$, RYOTARO OHKUMA $^{1,2,3}$, \\ MIDORI SHIDA $^{1,2}$, RIE ONOUE ${ }^{1,2}$, YUTARO KUBOTA $^{3}$, HIROTO MATSUI $^{3}$, \\ TOMOYUKI ISHIGURO ${ }^{3}$, YUYA HIRASAWA ${ }^{3}$, HIROTSUGU ARIIZUMI ${ }^{3}$, JUNJI TSURUTANI ${ }^{3,4}$, \\ KIYOSHI YOSHIMURA ${ }^{2,3}$, TAKUYA TSUNODA ${ }^{3}$, SHINICHI KOBAYASHI $^{2}$ and SATOSHI WADA ${ }^{1,2,3}$ \\ ${ }^{1}$ Department of Clinical Diagnostic Oncology, Clinical Research Institute for Clinical \\ Pharmacology and Therapeutics, Showa University, Tokyo, Japan; \\ ${ }^{2}$ Clinical Research Institute for Clinical Pharmacology and Therapeutics, Showa University, Tokyo, Japan; \\ ${ }^{3}$ Department of Medicine, Division of Medical Oncology, School of Medicine, Showa University, Tokyo, Japan; \\ ${ }^{4}$ Advanced Cancer Translational Research Institute, Showa University, Tokyo, Japan
}

\begin{abstract}
Background/Aim: Cancer immune therapy by immune checkpoint inhibitors (ICIs) is a promising therapeutic strategy for various cancer types. Among ICIs, anti-programmed cell death protein-1 (PDI) and antiprogrammed death-ligand 1 (PD-L1) antibodies have shown a remarkable clinical benefit. The present study aimed to address the functional and clinical significance of serum levels of soluble PD-L1 (sPD-L1) in patients. Materials and Methods: A total of 21 patients, 11 with NSCLC, nine with gastric cancer and one with bladder cancer, who underwent anti-PD-1 therapy were evaluated for $S P D-L 1$ concentration by ELISA analyses at diagnosis and after treatment. Results: Pretreatment levels of $S P D-L 1$ in patients who received ICIs were not remarkably correlated with the overall survival of these patients $(r=0.3394, p=0.1323)$. Reduction of plasma sPD-L1 level was significantly correlated with tumor regression in patients administered four cycles of treatment $(p<0.05)$. Conclusion: sPD-L1 might be derived and secreted from tumors and might be useful to identify primary responders to ICIs at a relatively early treatment timepoint.
\end{abstract}

*These Authors contributed equally to this study.

Correspondence to: Dr. Satoshi Wada, Department of Clinical Diagnostic Oncology, Clinical Research Institute for Clinical Pharmacology and Therapeutics, Showa University, 6-11-11 Kitakarasuyama, Setagaya-ku, Tokyo 157-8577, Japan. E-mail: stwada@med.showa-u.ac.jp

Key Words: Programmed death-ligand 1, immune checkpoint inhibitor.
Programmed death protein (PD1) and its ligand PD-L1 are central components in immune suppression, comprising the so-called 'immune checkpoint' $(1,2)$. Accumulating evidence revealed that the PD1/PD-L1 pathway is closely involved in resistance to antitumor immunity in multiple cancer types. Therefore, targeting immune checkpoints using immune checkpoint inhibitors (ICIs), such as those against PD1/PD-L1, has contributed to recent advances in cancer therapeutics $(3,4)$. However, many patients failed to respond or developed resistance after an initial response to ICIs. Predictive biomarkers for identifying potential responders to ICIs are currently under debate (5). Notably, several lines of evidence suggest a correlation between tumor PD-L1 expression and response to ICIs in variable malignancies including lung adenocarcinoma, melanoma, refractory Hodgkin's lymphoma, and other solid tumor types (6-10). Intriguingly, such correlation was reported for the expression level of membrane-bound PD-L1 (mPD-L1) on tumor tissues and plasma level of soluble PD-L1 (sPD-L1) in the blood of patients with cancer, highlighting the importance of their prognostic value. Several studies showed that high expression of sPD-L1 was associated with a poor prognosis in multiple types of malignant tumor. This suggests that SPDL1 might be a predictive marker for low treatment responses to conventional chemotherapy and patients with high expression of sPD-L1 might be suitable for ICI therapy (1120). However, it remains unclear whether the plasma sPDL1 level is derived from malignant tumors pre-existing in patients and reflects a potential response to ICIs. Here, we measured the plasma level of sPD-L1 collected from patients with non-small cell lung cancer (NSCLC) and those with gastric cancer who underwent anti-PD1 therapy and analyzed 
change in sPD-L1 expression from diagnosis to after treatment in order to address the clinical implications of measuring sPD-L1 in patients.

\section{Patients and Methods}

General information. We retrospectively analyzed data for 21 patients, 11 with first-line or previously treated NSCLC, nine with gastric cancer and one with bladder cancer, who received ICI treatment (240 mg nivolumab intravenously every 2 weeks, or 200 mg pembrolizumab intravenously every 3 weeks) at Showa University Hospital from January 2017 to April 2019. The patient characteristics, immunohistochemical assessment of PD-L1 in the tumor at the time of diagnosis, serum SPD-L1 concentration at the time of diagnosis and after 1-4 cycles of ICI treatment were evaluated. Relative change in SPD-L1 concentration was determined by dividing the sPD-L1 concentration after four cycles of ICI treatment by that at diagnosis. Overall survival (OS) referred to the time from diagnosis to the date of last follow-up or mortality. The efficacy of ICIs was assessed according to the Response Evaluation Criteria in Solid Tumors (RECIST), version 1.1 (21). Target lesions were assessed by computed tomography, and change in tumor size was defined as the percentage change in the tumor size from baseline to that after four cycles of ICI treatment. The control group for sPD-L1 consisted of samples collected from five healthy volunteers. The Ethics Committee of Showa University Hospital approved the study. All patients who participated in this research provided informed consent in participation.

PD-L1 determination. Formalin-fixed paraffin-embedded tissue samples were prepared from biopsy tissues of the patients before treatment. Immunohistochemical staining of mPD-L1 was performed by companion diagnostics according to the manufacturer's instructions: PD-L1 immunohistochemistry (IHC) 28-8 PharmaDX and PD-L1 IHC 22C3 PharmaDX were used before treatment with nivolumab and pembrolizumab (Dako, Glostrup, Denmark). Two investigators were blinded to the corresponding clinical outcome and independently evaluated specimens stained as serial sections. PD-L1 expression was quantitatively evaluated as tumor proportion score (TPS) according to the manufacturer's instructions.

Blood samples were collected before and after treatment, and the plasma levels of sPD-L1 were measured by enzyme-linked immunosorbent assay (ELISA) (DuoSet ELISA Development System Human PD-L1/B7-H1 and DuoSet Ancillary Reagent Kit 2; R\&D Systems, Inc., Minneapolis, MN, USA), according to the manufacturer's instructions. Standards and samples were prepared as follows. Recombinant human B7-H1 was diluted with 1\% Bovine serum albumin (BSA) in phosphate-buffered salts (PBS) for standards. Plasma was centrifuged and the supernatants were diluted 1:4 with $1 \%$ BSA as samples. A flat-bottom 96-well microplate was coated with $4.0 \mu \mathrm{g} / \mathrm{ml}$ mouse anti-human B7-H1 capture antibody in PBS, and the plate was sealed with an adhesive strip followed by overnight incubation. Thereafter, the plate was washed and blocked with $1 \%$ BSA in PBS for 1 hour. After washing, standards or samples were added to each well and the plate was sealed. Two hours after incubation, the plate was washed and then $50 \mathrm{ng} / \mathrm{ml}$ biotinylated goat anti-human B7-H1 detection antibody in PBS containing $1 \%$ BSA and $2.5 \%$ goat normal serum (R\&D Systems) was placed in each well. The plate was sealed and incubated for a further 2 hours. After washing, for colorimetric detection, streptavidin-horseradish peroxidase (1:200) was added to each well which was sealed and then incubated for 20 minutes avoiding direct light. After the plate was washed, a substrate solution consisting of a 1:1 mixture of $\mathrm{H}_{2} \mathrm{O}_{2}$ and tetramethylbenzidine was placed in each well and incubated for 20 minutes in a dark room. A termination solution was then added and the absorbance of each well was measured using a microplate reader (wavelength, 450 and $570 \mathrm{~nm}$; Powerscan HT; DS Pharma Biomedical Co., Ltd., Osaka, Japan). The reading at $570 \mathrm{~nm}$ was subtracted from that at $455 \mathrm{~nm}$ to correct for optical imperfections in the plate. Concentrations of sPDL1 were determined using a calibration curve. The minimum detectable concentration of SPD-L1 was $9.82 \mathrm{pg} / \mathrm{ml}$.

Data analysis. Statistical analysis was performed using Microsoft Excel (Microsoft Co., Redmond, WA, USA) and validity of the results was confirmed by JMP version 14.0 (SAS Institute, Cary, NC, USA). Data were analyzed using the unpaired Student's t-test. The results are presented as the mean \pm standard deviation. Linear correlation analysis was performed using Spearman's rank correlation. The comparisons of patient variables and SPD-L1 levels according to pretreatment levels were performed using Fisher's exact test. All tests were two-sided, and a $p$-value of less than 0.05 was considered statistically significant.

\section{Results}

Pretreatment levels of SPD-L1 in association with different clinicopathological characteristics and clinical responses. To explore whether there is an association between plasma SPDL1 concentration and clinicopathological characteristics, as well clinical responses, in patients on PD1 blockade therapy, we measured SPD-L1 concentrations at diagnosis and after four cycles of ICI treatment in plasma collected from 21 patients, 11 with NSCLC, nine with gastric cancer and one with bladder cancer. Four patients with NSCLC and nine with gastric cancer were treated with nivolumab; seven patients with NSCLC and 1 with bladder cancer were treated with pembrolizumab. The mean SPD-L1 level in the experimental group pre-ICI treatment was $469.7 \pm 298.8 \mathrm{pg} / \mathrm{ml}$ and was $323.0 \pm 108.4 \mathrm{pg} / \mathrm{ml}$ in the control group. In contrast, with previous reports $(12,14-16,18-20)$, there was no significant difference between the groups $(p=0.2969)$. We evaluated the potential association between pre-ICI treatment levels of SPDL1 and clinicopathological characteristics in the patients. For this purpose, we divided patients into two groups according to the median sPD-L1 level: low sPD-L1 $\leq 347.37 \mathrm{pg} / \mathrm{ml}$ and high PD-L1 $>347.37 \mathrm{pg} / \mathrm{ml}$. As shown in Table I, no significant correlation was observed between SPD-L1 level and patient's sex, age cancer type and pathological stage.

Detection of mPD-L1 expression in tumors was approved as a companion diagnostic to predict a responder to PD1/PDL1 blockade therapy. We assessed whether mPD-L1 expression, assessed by tumor IHC, routinely evaluated in 11 patients with NSCLC, was associated with SPD-L1 level. There was no significant correlation between mPD-L1 and 
Table I. Association of clinical factors with plasma level of soluble programmed death-ligand 1 (sPD-L1). Patients were divided according to the median $S P D-L 1$ level.

\begin{tabular}{|c|c|c|c|c|}
\hline \multirow[b]{2}{*}{ Variable } & \multirow[b]{2}{*}{ All, n (\%) } & \multicolumn{2}{|c|}{ sPD-L1, n (\%) } & \multirow[b]{2}{*}{$p$-Value } \\
\hline & & $\begin{array}{c}\text { Low } \\
(\leq 347.37 \\
\mathrm{pg} / \mathrm{ml})\end{array}$ & $\begin{array}{c}\text { High } \\
(>347.37 \\
\text { pg/ml })\end{array}$ & \\
\hline \multicolumn{5}{|l|}{ Gender } \\
\hline Male & $15(71.4)$ & $8(72.7)$ & $7(70.0)$ & $>0.99$ \\
\hline Female & $6(28.6)$ & $3(27.3)$ & $3(30.0)$ & \\
\hline \multicolumn{5}{|l|}{ Age } \\
\hline$<70$ Years & $12(57.1)$ & 7 (63.6) & $5(50.0)$ & 0.6699 \\
\hline$\geq 70$ Years & $9(42.9)$ & $4(36.4)$ & $5(50.0)$ & \\
\hline \multicolumn{5}{|l|}{ Cancer type } \\
\hline NSCLC & $11(52.4)$ & $5(45.5)$ & $6(60.0)$ & 0.6699 \\
\hline Other & $10(47.6)$ & $6(54.5)$ & $4(40.0)$ & \\
\hline \multicolumn{5}{|c|}{ Clinical stage } \\
\hline III & $6(28.6)$ & $3(27.3)$ & $3(30.0)$ & 0.6327 \\
\hline IV & $15(71.4)$ & $8(72.7)$ & $7(70.0)$ & \\
\hline
\end{tabular}

NSCLC: Non-small cell lung cancer.

sPD-L1 levels $(\mathrm{r}=0.1955, p=0.5645)$. Then whether expression of mPD-L1 and sPD-L1 pre ICI treatment correlated with patient outcome was assessed because a previous report showed mPD-L1 expression determined by tumor IHC was associated with a poor prognosis in various cancer types (22). Furthermore, pretreatment serum levels of sPD-L1 in patients who received conventional chemotherapy other than ICIs were positively correlated with a poorer prognosis (11-20). In contrast to these previous studies, mPD-L1 expression as well as pretreatment levels of sPDL1 in patients who received ICIs were not significantly correlated with the OS of these patients ( $r=-0.1981$, $p=0.5592$ and $\mathrm{r}=0.3394, p=0.1323$, respectively). The clinicopathological characteristics including the sPD-L1 concentration as well as OS are summarized in Table II.

Association between SPD-L1 levels and tumor regression after four cycles of ICI treatment. Because the SPD-L1 levels pre-ICI treatment appeared to have no prognostic significance before PD1 blockade therapy, it prompted us to investigate whether a change in SPD-L1 level would be observed in response to ICI treatment. For this purpose, we calculated changes in SPD-L1 concentration from baseline to after four cycles of ICI treatment and evaluated its potential association with OS, mPD-L1 expression and percentage change of tumor size. Among 21 patients, data for nine were available who had been administered more than four cycles ICI treatment. Interestingly, the change in sPD-L1 concentration was significantly correlated with the

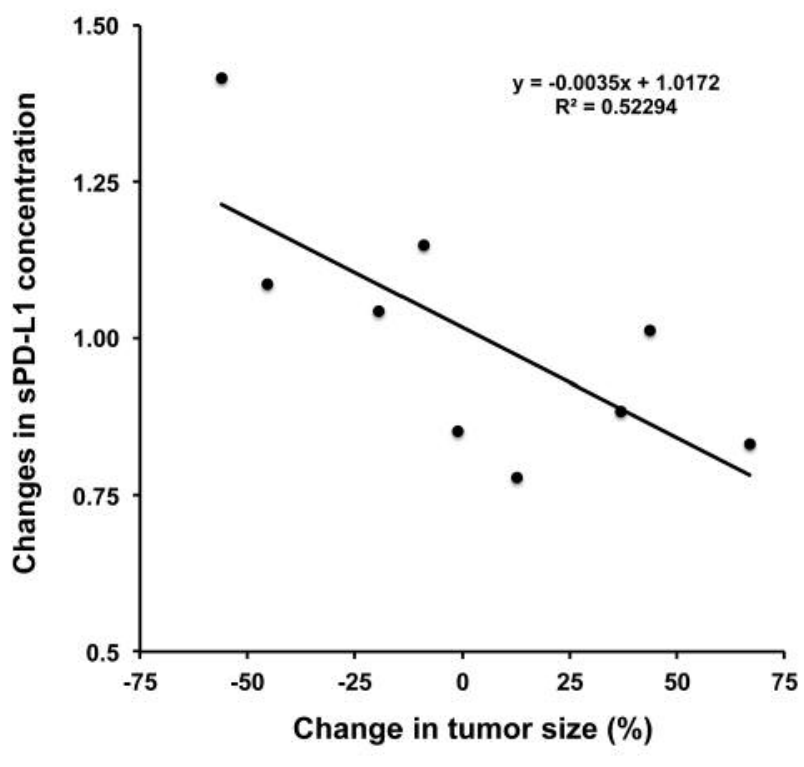

Figure 1. Linear correlation between change in serum level of soluble programmed death-ligand $1(s P D-L 1)$ and tumor regression. Relative change in serum $S P D-L 1$ was calculated as the concentration after four cycles of immune checkpoint inhibitor therapy divided by that by that at diagnosis. Percentage change in the tumor size after four cycles of ICI treatment was calculated relative to that at baseline. A decrease in SPD-L1 concentration from pre to post immune checkpoint inhibitor treatment was significantly correlated with reduction of tumor size $(r=0.7231, p=0.0277)$.

percentage change of tumor size $(\mathrm{r}=0.7231, p=0.0277$; Figure 1), but not with OS ( $\mathrm{r}=-0.3211, p=0.3995)$ or mPDL1 expression $(\mathrm{r}=0.4045, p=0.4262)$. In addition, as shown in Figure 2, the patients of this study that showed the greatest tumor regression and progression in response to ICIs, case 3 and case 14, respectively (Figure 2A), had similar levels of sPD-L1 in response to four cycles of ICI, but remarkably different levels pre-ICI therapy (Figure 2B). Table II summarizes the clinicopathological characteristics including the change of plasma sPD-L1 concentration from pre to post ICI treatment (four cycles), as well as the OS of nine patients for whom sPD-L1 levels at post four cycles of ICI were available.

\section{Discussion}

Recently, the presence of mPD-L1 expression in tumors has been used clinically to predict ICI responders: Clones 28-8, 22C3 and SP142 have been approved as companion diagnostic antibodies for nivolumab, pembrolizumab and atezolizumab, respectively (23). However, not all patients who present with high levels of mPD-L1 expression respond to ICI treatment, and therefore attempts to establish new 
A

Case 3

Case14
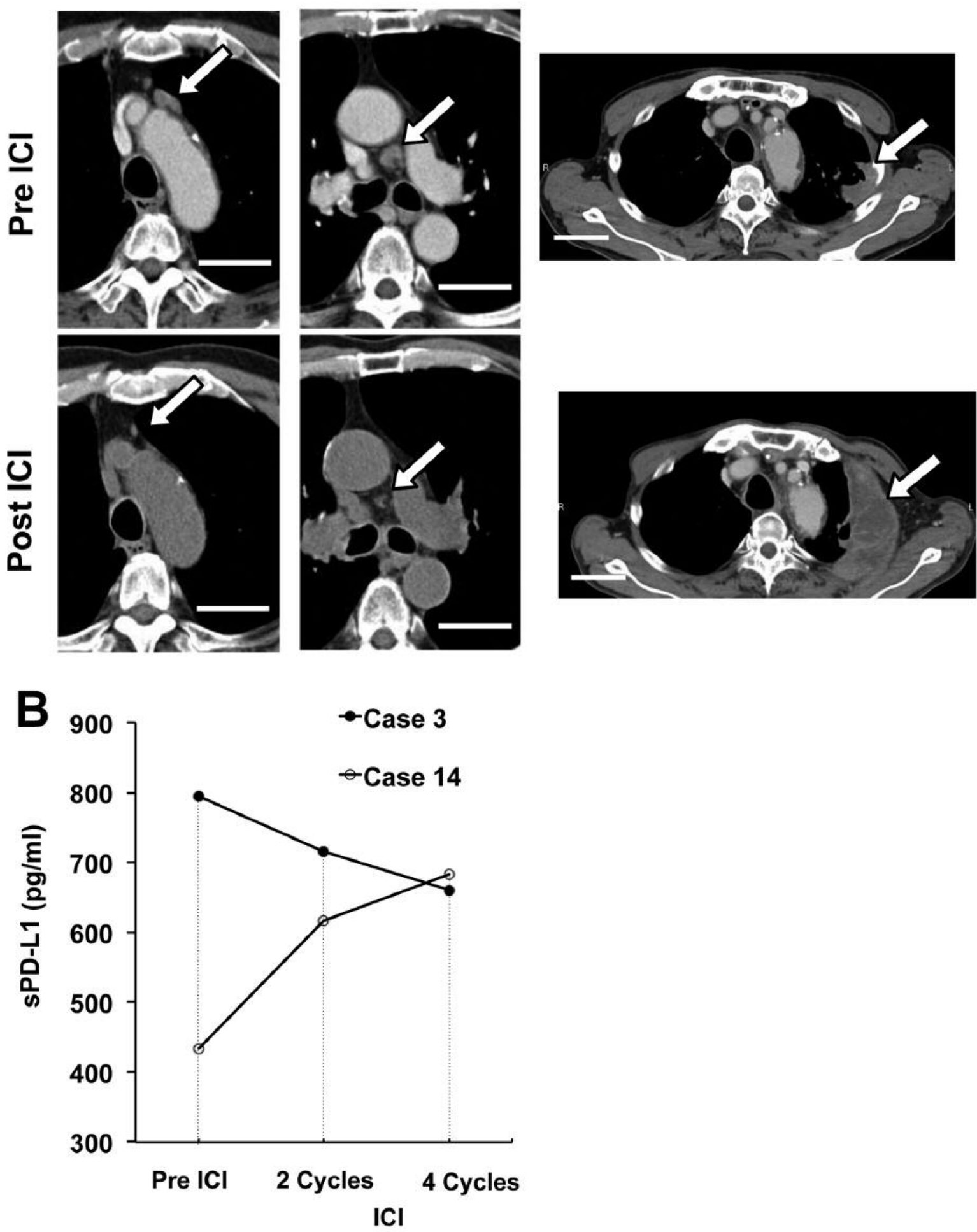

Figure 2. Changes in tumor size and serum level of soluble programmed death-ligand 1 (sPD-L1) level during treatment. A: Representative computed tomographic images of cases 3 (greatest tumor regression) and 14 greatest tumor progression) at baseline (upper panel) and after four cycles of treatment with immune checkpoint inhibitor (lower panel). White arrows indicate measurable and assessable lesions. Bars=5 cm. B: sPD-L1 concentration before and during immune checkpoint inhibitor treatment. Case 3 was treated with nivolumab and case 14 was treated with pembrolizumab. 
Table II. Clinicopathological features, plasma soluble programmed death-ligand 1 (sPD-L1) concentration, tumor regression and overall survival (OS) for all study patients.

\begin{tabular}{|c|c|c|c|c|c|c|c|c|c|c|c|}
\hline \multirow[b]{2}{*}{$\begin{array}{l}\text { Case } \\
\text { no. }\end{array}$} & \multirow[b]{2}{*}{ Gender } & \multirow[b]{2}{*}{$\begin{array}{l}\text { Age, } \\
\text { years }\end{array}$} & \multirow[b]{2}{*}{$\begin{array}{l}\text { Cancer } \\
\text { type }\end{array}$} & \multirow[b]{2}{*}{ Stage } & \multirow[b]{2}{*}{ ICI } & \multirow[b]{2}{*}{$\begin{array}{c}\text { Tumor } \\
\text { mPD-L1 } \\
\text { IHC (\%) }\end{array}$} & \multicolumn{3}{|c|}{ sPD-L1 } & \multirow[b]{2}{*}{$\begin{array}{l}\text { Relative change } \\
\text { in tumor } \\
\text { size }(\%)^{*}\end{array}$} & \multirow[b]{2}{*}{$\begin{array}{c}\text { OS } \\
\text { (days) }\end{array}$} \\
\hline & & & & & & & Pre-ICI & Post-ICI* & $\begin{array}{l}\text { Relative } \\
\text { change* }\end{array}$ & & \\
\hline 1 & M & 78 & NSCLC & IV & Nivolumab & $20-30$ & 347.37 & 306.93 & 0.88 & 36.9 & 828 \\
\hline 2 & $\mathrm{~F}$ & 70 & NSCLC & IVB & Nivolumab & $50-60$ & 844.57 & 881.25 & 1.04 & -19.4 & 169 \\
\hline 3 & M & 67 & NSCLC & IIIA $^{\mathrm{R}}$ & Nivolumab & $70-80$ & 794.43 & 660.80 & 0.83 & 66.9 & 694 \\
\hline 4 & M & 63 & GC & IIIA $^{R}$ & Nivolumab & N/A & 251.85 & 273.67 & 1.09 & -45.3 & 126 \\
\hline 5 & M & 74 & GC & IV & Nivolumab & N/A & 258.06 & N/A & N/A & N/A & 136 \\
\hline 6 & M & 68 & GC & IV & Nivolumab & N/A & 417.30 & 479.23 & 1.15 & -9.0 & 249 \\
\hline 7 & M & 67 & NSCLC & IIIA & Nivolumab & $50-60$ & 363.79 & 283.11 & 0.78 & 12.7 & 247 \\
\hline 8 & $\mathrm{~F}$ & 68 & GC & IV & Nivolumab & N/A & 597.48 & 509.10 & 0.85 & -1.1 & 70 \\
\hline 9 & M & 66 & GC & IIIB & Nivolumab & N/A & 337.58 & N/A & N/A & N/A & 221 \\
\hline 10 & M & 60 & GC & $\mathrm{IIIB}^{\mathrm{R}}$ & Nivolumab & N/A & 798.41 & N/A & N/A & N/A & 44 \\
\hline 11 & $\mathrm{~F}$ & 49 & GC & IIIB & Nivolumab & N/A & 295.77 & N/A & N/A & N/A & 75 \\
\hline 12 & $\mathrm{~F}$ & 75 & GC & IV & Nivolumab & N/A & 283.11 & N/A & N/A & N/A & 257 \\
\hline 13 & $\mathrm{~F}$ & 57 & GC & IV & Nivolumab & N/A & 300.55 & N/A & N/A & N/A & 46 \\
\hline 14 & M & 72 & NSCLC & IV & Pembrolizumab & $70-80$ & 331.09 & 468.78 & 1.42 & -56.0 & 217 \\
\hline 15 & M & 71 & NSCLC & IV & Pembrolizumab & $60-70$ & 1491.14 & N/A & N/A & N/A & 679 \\
\hline 16 & M & 59 & NSCLC & IV & Pembrolizumab & $60-70$ & 284.68 & N/A & N/A & N/A & 46 \\
\hline 17 & M & 64 & NSCLC & IV & Pembrolizumab & $60-70$ & 253.40 & 256.50 & 1.01 & 43.6 & 644 \\
\hline 18 & M & 70 & NSCLC & IV & Pembrolizumab & $70-80$ & 392.04 & N/A & N/A & N/A & 51 \\
\hline 19 & M & 71 & NSCLC & IV & Pembrolizumab & 90 & 487.97 & N/A & N/A & N/A & 137 \\
\hline 20 & $\mathrm{~F}$ & 70 & BLDC & IV & Pembrolizumab & N/A & 442.88 & N/A & N/A & N/A & 402 \\
\hline 21 & M & 68 & NSCLC & IVB & Pembrolizumab & $10-20$ & 289.43 & N/A & N/A & N/A & 162 \\
\hline
\end{tabular}

F: Female; GC: gastric cancer; ICI: immune checkpoint inhibitor; IHC: immunohistochemistry; M: male; mPD-L1: membranous PD-L1; NSCLC: non-small cell lung cancer; R: recurrence. *Relative change in serum sPD-L1 was calculated as the concentration after four cycles of ICI therapy divided by that at diagnosis. Percentage change in the tumor size after four cycles of ICI treatment was calculated relative to that at baseline.

companion diagnostic biomarkers using liquid biopsy are underway (24). In line with this, increasing numbers of studies have suggested that the plasma level of sPD-L1 expression is associated with tumor aggressiveness and shorter patient survival, and thus might be a predictive biomarker to identify ICI responders (11-20). To date, it is still unclear whether ICI treatment-dependent fluctuations of sPD1 level occur during treatment cycles. In this study, we demonstrated, for the first time, that reduction of sPD-L1 level after four cycles of ICI treatment did not correlate with OS, but significantly correlated with tumor regression in patients with NSCLC and gastric cancer. This result indicated that the SPD-L1 level might reflect the total active cancer cells in an individual, and therefore might be linked to the size of lesions evaluable for ICI response. Notably, these observations might address one of the unresolved questions of where sPD-L1 is derived from. Although treatment associated-tumor disruption should increase tumorderived PD-L1, including mPD-L1, we observed that SPDL1 significantly decreased in association with regression of the tumor. Thus, we presumed that SPD-L1 might not be derived from tumors disrupted by ICI treatment but mainly secreted from viable tumors. Our notion is supported by previous reports showing that SPD-L1 levels in patients with lymphoma were reduced by treatment with conventional chemotherapeutic agents $(18,19)$. Furthermore, it was reported that a secreted form of PD-L1 derived from alternative splicing might be involved in therapeutic resistance to ICIs in malignant melanoma (25). Notedly, Gong et al. identified other forms of sPD-L1 splice variants that might act as decoys, resulting in resistance to PD-L1 blockade therapy. In addition, levels of sPD-L1 in patients harboring sPD-L1 splice variants were much higher than in healthy controls and patients without sPD-L1 splice variants (26). Taken together with our finding, this shows that sPD$\mathrm{L} 1$, to which resistance to ICIs such as anti-PD-L1 is attributed, might be secreted by tumors and therefore might be a useful marker when combined with RECIST for estimating the volume of viable cancer in patients. In addition, patients whose levels of sPD-L1 decreased after four cycles of ICI treatment might be primary responders for ICI therapy irrespective of their OS. However, in conflict with several previous reports $(12,14-16,18-20)$, there was no significant difference in SPD-L1 levels between healthy 
volunteers and patients with regard to our experimental settings. This conflict might be causally related to antibody specificity, especially when detecting SPD-L1 splice variants. However, the functional role as well as clinicopathological significance of the secreted SPD-L1 splice variants is still enigmatic. Further improvements to the detection system to distinguish between SPD-L1 variants are needed.

In summary, although our study had a low sample size, a significant correlation between a reduction of SPD-L1 levels and tumor regression was observed after four cycles of ICI treatment in patients with NSCLC and gastric cancer. Therefore, our data suggest that SPD-L1 might be secreted from tumors and be useful for evaluating primary responders to ICIs at a relatively early time point during treatment cycles. Importantly, our samples were collected from patients administered PD1 blockade agents, nivolumab and pembrolizumab. However, it is still unknown how the use of PD-L1 blockade agents affects the PD-L1 level. New insights might be provided regarding the clinical significance of determining the SPD-L1 level to investigate whether there is a differential effect on SPD-L1 level arising from use of anti-PD1 or anti-PD-L1 agents.

\section{Conflicts of Interest}

None of the Authors declares any conflict of interest.

\section{Authors' Contributions}

$\mathrm{KH}$ and SW conceived and designed this study. KH, RO, MS, RO, YK, HM, TI, YH, HA, TA and SW collected samples and recorded the general data and the observation indicators of patients. MW performed all ELISAs. KA analyzed the data and wrote the first draft of the article. JT, KY, TT, SK and SW critically reviewed and corrected the article. All Authors reviewed and approved the final version of the article.

\section{Acknowledgements}

The Authors thank Edanz Group (www.edanzediting.com/ac) for editing a draft of this article.

\section{References}

1 Freeman GJ, Long AJ, Iwai Y, Bourque K, Chernova T, Nishimura H, Fitz LJ, Malenkovich N, Okazaki T, Byrne MC, Horton HF, Fouser L, Carter L, Ling V, Bowman MR, Carreno BM, Collins M, Wood CR and Honjo T: Engagement of the PD1 immunoinhibitory receptor by a novel b7 family member leads to negative regulation of lymphocyte activation. J Exp Med 192(7): 1027-1034, 2000. PMID: 2193311. DOI: 10.1084/jem. 192.7.1027

2 Carreno BM and Collins M: The B7 family of ligands and its receptors: New pathways for costimulation and inhibition of immune responses. Annu Rev Immunol 20: 29-53, 2002. DOI: 10.1146/annurev.immunol.20.091101.091806
3 Zou W, Wolchok JD and Chen L: PD-L1 (B7-H1) and PD1 pathway blockade for cancer therapy: Mechanisms, response biomarkers, and combinations. Sci Transl Med 8(328): 328rv324, 2016. PMID: 4859220. DOI: 10.1126/scitranslmed. aad7118

4 Hamanishi J, Mandai M, Matsumura N, Abiko K, Baba T and Konishi I: Pd-1/pd-11 blockade in cancer treatment: Perspectives and issues. Int J Clin Oncol 21(3): 462-473, 2016. PMID: 4901122. DOI: $10.1007 / \mathrm{s} 10147-016-0959-\mathrm{Z}$

5 Meng X, Huang Z, Teng F, Xing L and Yu J: Predictive biomarkers in PD1/PD-L1 checkpoint blockade immunotherapy. Cancer Treat Rev 41(10): 868-876, 2015. PMID: 26589760. DOI: $10.1016 /$ j.ctrv.2015.11.001

6 Wang Q, Liu F and Liu L: Prognostic significance of PD-L1 in solid tumor: An updated meta-analysis. Medicine 96(18): e6369, 2017. PMID: 5419898. DOI: 10.1097/MD.0000000000006369

7 Robert C, Long GV, Brady B, Dutriaux C, Maio M, Mortier L, Hassel JC, Rutkowski P, McNeil C, Kalinka-Warzocha E, Savage KJ, Hernberg MM, Lebbe C, Charles J, Mihalcioiu C, Chiarion-Sileni V, Mauch C, Cognetti F, Arance A, Schmidt H, Schadendorf D, Gogas H, Lundgren-Eriksson L, Horak C, Sharkey B, Waxman IM, Atkinson V and Ascierto PA: Nivolumab in previously untreated melanoma without BRAF mutation. N Engl J Med 372(4): 320-330, 2015. DOI: 10.1056/NEJMoa1412082

8 Ansell SM, Lesokhin AM, Borrello I, Halwani A, Scott EC, Gutierrez M, Schuster SJ, Millenson MM, Cattry D, Freeman GJ, Rodig SJ, Chapuy B, Ligon AH, Zhu L, Grosso JF, Kim SY, Timmerman JM, Shipp MA and Armand P: Pd-1 blockade with nivolumab in relapsed or refractory Hodgkin's lymphoma. N Engl J Med 372(4): 311-319, 2015. PMID: 4348009. DOI: 10.1056/NEJMoa1411087

9 Fehrenbacher L, Spira A, Ballinger M, Kowanetz M, Vansteenkiste J, Mazieres J, Park K, Smith D, Artal-Cortes A, Lewanski C, Braiteh F, Waterkamp D, He P, Zou W, Chen DS, Yi J, Sandler A, Rittmeyer A and Group PS: Atezolizumab versus docetaxel for patients with previously treated non-smallcell lung cancer (POPLAR): A multicentre, open-label, phase 2 randomised controlled trial. Lancet 387(10030): 1837-1846, 2016. PMID: 26970723. DOI: 10.1016/S0140-6736(16)00587-0

10 Herbst RS, Baas P, Kim DW, Felip E, Perez-Gracia JL, Han JY, Molina J, Kim JH, Arvis CD, Ahn MJ, Majem M, Fidler MJ, de Castro G Jr., Garrido M, Lubiniecki GM, Shentu Y, Im E, Dolled-Filhart $M$ and Garon EB: Pembrolizumab versus docetaxel for previously treated, PD-L1-positive, advanced nonsmall-cell lung cancer (KEYNOTE-010): A randomised controlled trial. Lancet 387(10027): 1540-1550, 2016. PMID: 26712084. DOI: 10.1016/S0140-6736(15)01281-7

11 Okuma Y, Hosomi Y, Nakahara Y, Watanabe K, Sagawa Y and Homma S: High plasma levels of soluble programmed cell death ligand 1 are prognostic for reduced survival in advanced lung cancer. Lung Cancer 104: 1-6, 2017. PMID: 28212990. DOI: 10.1016/j.lungcan.2016.11.023

12 Nagato T, Ohkuri T, Ohara K, Hirata Y, Kishibe K, Komabayashi Y, Ueda S, Takahara M, Kumai T, Ishibashi K, Kosaka A, Aoki N, Oikawa K, Uno Y, Akiyama N, Sado M, Takei H, Celis E, Harabuchi Y and Kobayashi H: Programmed death-ligand 1 and its soluble form are highly expressed in nasal natural killer/Tcell lymphoma: A potential rationale for immunotherapy. Cancer Immunol Immunother 66(7): 877-890, 2017. PMID: 28349165. DOI: $10.1007 / \mathrm{s} 00262-017-1987-\mathrm{x}$ 
13 Takahashi N, Iwasa S, Sasaki Y, Shoji H, Honma Y, Takashima A, Okita NT, Kato K, Hamaguchi T and Yamada Y: Serum levels of soluble programmed cell death ligand 1 as a prognostic factor on the first-line treatment of metastatic or recurrent gastric cancer. J Cancer Res Clin Oncol 142(8): 1727-1738, 2016. PMID: 27256004. DOI: 10.1007/s00432-016-2184-6

14 Finkelmeier F, Canli O, Tal A, Pleli T, Trojan J, Schmidt M, Kronenberger B, Zeuzem S, Piiper A, Greten FR and Waidmann O: High levels of the soluble programmed death-ligand (sPDL1) identify hepatocellular carcinoma patients with a poor prognosis. Eur J Cancer 59: 152-159, 2016. PMID: 27039170 DOI: 10.1016/j.ejca.2016.03.002

15 Rossille D, Gressier M, Damotte D, Maucort-Boulch D, Pangault C, Semana G, Le Gouill S, Haioun C, Tarte K, Lamy T, Milpied N, Fest T, Groupe Ouest-Est des Leucemies et Autres Maladies du S and Groupe Ouest-Est des Leucemies et Autres Maladies du S: High level of soluble programmed cell death ligand 1 in blood impacts overall survival in aggressive diffuse large b-cell lymphoma: Results from a French multicenter clinical trial. Leukemia 28(12): 2367-2375, 2014. PMID: 24732592. DOI: 10.1038/leu.2014.137

16 Zhang J, Gao J, Li Y, Nie J, Dai L, Hu W, Chen X, Han J, Ma $X$, Tian G, Wu D, Shen L and Fang J: Circulating PD-L1 in NSCLC patients and the correlation between the level of PD-L1 expression and the clinical characteristics. Thorac Cancer 6(4): 534-538, 2015. PMID: 4511334. DOI: 10.1111/1759-7714.12247

17 Chang B, Huang T, Wei H, Shen L, Zhu D, He W, Chen Q, Zhang H, Li Y, Huang R, Li W and Wu P: The correlation and prognostic value of serum levels of soluble programmed death protein 1 (sPD1) and soluble programmed death-ligand 1 (sPDL1) in patients with hepatocellular carcinoma. Cancer Immunol Immunother 68(3): 353-363, 2019. PMID: 6426820. DOI: $10.1007 / \mathrm{s} 00262-018-2271-4$

18 Shen H, Ji Y, Zhou D, Zhang Y, Wang W, Sun J and Zhang W: Soluble programmed death-ligand 1 are highly expressed in peripheral T-cell lymphoma: A biomarker for prognosis. Hematology 24(1): 392-398, 2019. PMID: 30890046. DOI: 10.1080/16078454.2019.1590965

19 Yang $\mathrm{J}$ and Hu G: Significance of PD-L1 in the diagnosis and treatment of B-cell malignant lymphoma. Oncol Lett 17(3): 3382-3386, 2019. PMID: 6396271. DOI: 10.3892/ol.2019.9982
20 Wang L, Wang H, Chen H, Wang WD, Chen XQ, Geng QR, Xia $\mathrm{ZJ}$ and Lu Y: Serum levels of soluble programmed death ligand 1 predict treatment response and progression-free survival in multiple myeloma. Oncotarget 6(38): 41228-41236, 2015. PMID: 4747402. DOI: 10.18632/oncotarget.5682

21 Eisenhauer EA, Therasse P, Bogaerts J, Schwartz LH, Sargent D, Ford R, Dancey J, Arbuck S, Gwyther S, Mooney M, Rubinstein L, Shankar L, Dodd L, Kaplan R, Lacombe D and Verweij J: New response evaluation criteria in solid tumours: Revised RECIST guideline (version 1.1). Eur J Cancer 45(2): 228-247, 2009. PMID: 19097774. DOI: 10.1016/j.ejca.2008. 10.026

22 Kythreotou A, Siddique A, Mauri FA, Bower M and Pinato DJ: PD-L1. J Clin Pathol 71(3): 189-194, 2018. PMID: 29097600. DOI: $10.1136 /$ jclinpath-2017-204853

23 Fujimoto D, Sato Y, Uehara K, Ishida K, Fukuoka J, Morimoto T, Kawachi H, Mori R, Ito M, Teraoka S, Nagata K, Nakagawa A, Otsuka K, Imai Y and Tomii K: Predictive performance of four programmed cell death ligand 1 assay systems on nivolumab response in previously treated patients with non-small cell lung cancer. J Thorac Oncol 13(3): 377-386, 2018. PMID: 29233789. DOI: $10.1016 /$ j.jtho.2017.11.123

24 Cyriac G and Gandhi L: Emerging biomarkers for immune checkpoint inhibition in lung cancer. Semin Cancer Biol 52(Pt 2): 269-277, 2018. PMID: 29782924. DOI: 10.1016/j.semcancer. 2018.05.006

25 Zhou J, Mahoney KM, Giobbie-Hurder A, Zhao F, Lee S, Liao X, Rodig S, Li J, Wu X, Butterfield LH, Piesche M, Manos MP, Eastman LM, Dranoff G, Freeman GJ and Hodi FS: Soluble PDL1 as a biomarker in malignant melanoma treated with checkpoint blockade. Cancer Immunol Res 5(6): 480-492, 2017. PMID: 5642913. DOI: 10.1158/2326-6066.CIR-16-0329

26 Gong B, Kiyotani K, Sakata S, Nagano S, Kumehara S, Baba S, Besse B, Yanagitani N, Friboulet L, Nishio M, Takeuchi K, Kawamoto H, Fujita N and Katayama R: Secreted PD-L1 variants mediate resistance to pd-11 blockade therapy in nonsmall cell lung cancer. J Exp Med 216(4): 982-1000, 2019. PMID: 6446862. DOI: 10.1084/jem.20180870

Received July 15, 2019

Revised July 28, 2019

Accepted July 30, 2019 